\title{
Resinas utilizadas en la confección de prótesis provisionales. Reporte de un caso.
}

Resins used in the manufacture of pro-

visional prothesis. Case report.
As resinas utilizadas na fabricação de

temporários. Reporte de um caso.
Juan José Christiani. Docente Auxiliar $I^{\circ}$ categoría. Cátedra

Preclínica de Prótesis. jjchristiani@odn.unne.edu.ar

José Rafael Devecchi. Profesor Adjunto a/c Cátedra Preclínica de Prótesis.

María Eugenia Zamudio Profesora Titular Cátedra de Biomateriales.

Lugar de Trabajo Facultad de Odontología Universidad Nacional del Nordeste Av. Libertad 5450. CP.3400. Corrientes. Argentina

\section{Resumen}

Las restauraciones provisionales son aquellas que se instalan en la boca antes de las restauraciones definitivas por lo tanto debe permanecer en boca el tiempo que demande la confección de la definitiva. Además de todas las necesidades funcionales requeridas del material provisional deben cumplir requisitos estéticos ya que han pasado a ser un factor de grandes demandas $y$ exigencias por parte de los pacientes. Las restauraciones provisionales utilizadas en prótesis fija han cambiado y evolucionado en los últimos años como consecuencia de los recientes avances en odontología restauradora

En la actualidad la posibilidad de elección de resinas para provisionales en el mercado son diversas. Cada material presenta sus ventajas y desventajas. Mientras que los materiales tradicionales todavía están en uso hoy, los nuevos materiales propuestos van actualizando y mejorando sus propiedades, tal es el caso de las resinas bisacrílicas de reciente aparición que han ayudado a eliminar algunos de los problemas asociados con los tradicionales materiales acrílicos.

En el presente artículo se hacer referencia a un caso clínico de confección de provisionales utilizando como material resinas bis-acrílicas por la técnica directa.

\section{Palabras claves}

Prótesis provisional, restauración provisional, resinas acrílicas, resinas bisacrílicas. 


\section{Summary}

Provisional restorations are those that are installed in the mouth before the definitive restorations should therefore remain in mouth the time that require the making of the final. Besides all the functional needs required provisional material must meet aesthetic requirements as they have become a major factor demands and aesthetic requirements. Provisional restorations in fixed prosthesis used have changed and evolved over the years as a result of recent advances in restorative dentistry.

At present, the choice of provisional resins on the market are diverse. Each material has its advantages and disadvantages. While traditional materials are still in use today, the materials will update and improve their properties as in the case of bis-acrylic resins for recently introduced have helped eliminate some of the problems associated with traditional acrilycs.

This articule presents a case of the making of provisional bis-acrylic resins for the direct technique.

\section{Keywords}

Provisional prosthesis, bisacrílicas resins,.tempory restoration-acrylic resins.

\section{Resumo}

Restaurações provisórias são aqueles que são instalados na boca antes de as restaurações definitivas deverão, por conseguinte, permanecem no tempo que demora a tornar boca final. Além de todas as necessidades funcionais necessitavam de um material provisório devem atender aos requisitos estéticos, eles tornaram-se um fator importante demandas e exigências estéticas. Restaurações provisórias em prótese fixa utilizada mudaram e evoluíram ao longo dos anos, como resultado dos recentes avanços em odontologia restauradora.

Hoje, a escolha de resinas para o mercado interino são diversas. Cada material tem suas vantagens e desvantagens. Enquanto os materiais tradicionais ainda estão em uso hoje em dia, os materiais irão actualizar e melhorar as suas propriedades, como no caso de resinas bis-acrílico introduzido recentemente para ter ajudado a eliminar alguns dos problemas associados com os acrílicos tradicionais.

Este artigo apresenta um caso de a preparação de intermediários bis acrílicos resinas para a técnica direta.

\section{Palavras chave}

Prótese provisória, restauração provisória, resinas acrílicas, bis acrílico.

\section{Introducción}

Las prótesis provisionales son aquellas que colocamos en la boca del paciente para evitar que éste quede desdentado mientras no esté su prótesis definitiva. El término provisional significa que se establece por un período, pendiente de una restauración definitiva'. Su correcta fabricación tiene gran implicancia en el éxito o el fracaso del tratamiento definitivo. Protege el diente y evita la exposición de dentina, y en consecuencia que la pulpa sea afectada, además actúa como aislante térmico ${ }^{2-5}$.

Las prótesis provisionales sirven de mock-up o maqueta, y constituyen un factor de predictibilidad de la futura rehabilitación porque permiten ver si el tratamiento futuro se ajustará a los requerimientos del paciente.

Por otro lado, al profesional le sirve para ver la adaptabilidad de las diferentes estructuras y para valorar la necesidad o no de modificar el plan de tratamiento.

Uno de los inconvenientes que presentan estos materiales es el cambio de color, al estar en contacto con sustancias pigmentantes ${ }^{5}$. Esto es particularmente problemático cuando las restauraciones provisionales son sometidas a exposición prolongada a los colorantes durante el tratamiento prolongado6, produciendo como consecuencia, la insatisfacción del paciente y un gasto adicional para el reemplazo.

En los tratamientos de rehabilitación del sector anterior es importante cuidar mucho la estética y naturalidad de los dientes; por ello, en muchas ocasiones, se decide el material que se va a utilizar en función de su estabilidad en el color ${ }^{7}$.

Los cambios del color de los materiales provisionales se han valorado en muchos estudios utili-

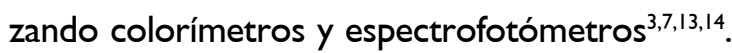
Estos instrumentos utilizan el sistema de color CIELAB, creado en 1978 por la Commission 
Intérnationale de l'Éclairage para determinar el color basándose en la percepción humana ${ }^{5}$.

El odontólogo debe afrontar a diario nuevos desafíos debido al aumento del nivel de exigencia estética de los pacientes y teniendo en cuenta que en la actualidad el mercado ofrece una serie de opciones, (que buscan principalmente simplificar la técnica, con muy buenos resultados), se hace necesario profundizar en el conocimiento de los materiales disponibles para la confección de prótesis provisionales.

La oferta de materiales dentales para la confección personalizada de coronas provisionales hace que haya materiales que faciliten la confección de la misma con una alta estética ${ }^{8}$.

Sin embargo los de mayor éxito en el mercado se pueden clasificar en dos grupos principales en relación a su estructura química que a su vez estos varían por las propiedades de fraguado y por el tipo de manipulación 7 :

-Sistemas polvo-líquido basados en polimetacrilatol metilmetacrilato (PMNA/MMA) o bien basados en metacrilatos de mayor peso molecular como el polietilmetacrilato (PEMA) o el metacrilato de isobutilo.

-Sistema pasta-pasta basados en resinas bis-acrílicas ${ }^{7}$.

Los sistemas PMNA Y PEMA vienen en proporción polvo líquido y necesita un mezclado manual. Pueden usarse para provisionales únicos como múltiples, son de relativo bajo costo y de fácil pulido. Poseen una temperatura de exotermia que debe ser tenido en cuenta para no producir daño pulpar ${ }^{14}$. Estos materiales deben ser rebasados antes de su fijación para un correcto sellado marginal ${ }^{9}$.

Las resinas acrílicas se han utilizado durante años como materiales provisionales de elección, ya que mostraban buenas propiedades y un bajo costo.

Pero en la actualidad, las resinas bisacrílicas se emplean con mayor frecuencia, dadas sus buenas propiedades mecánicas y su facilidad de confección. Poseen resistencia a la rotura, característica clave de la durabilidad de un provisional que se comprobó en estudios de cargas masticatorias ${ }^{8}$. A su vez, la dureza superficial de los provisionales confeccionados con resinas bis acrílicas es mayor que la confeccionados con PMMA. Esto estaría dado en la capacidad de establecer enlaces cruzados que tienen los acrílicos bifuncionales. El propio relleno que contiene incrementa la resistencia al desgaste.

La dureza de la mayoría de los materiales disminuye con el tiempo, sin embargo las resinas bis acrílicas exhiben una dureza superior a las resinas de $M_{M}{ }^{6}$. Hay menos riesgo de daño a la pulpa con bis-acryls ya que estos materiales típicamente generan mucho menos calor durante el proceso de polimerización?.

Como ventajas las resinas Bis-acrilicas se puede mencionar:

- Fáciles de usar (la mayoría se presenta en cartucho de automezcla con pistola, y también en jeringas individuales).

- No necesitan pulido.

- Reacción de fraguado con poca exotérmica.

- Se pueden reparar mediante composite o resina fluida.

- La contracción de polimerización es baja (menor a un 3\%).

Como desventajas:

- Poca estabilidad de color (en ciertas marcas) $)^{8}$

- Las resinas bis-acrílicas se pueden romper con relativa facilidad cuando se colocan en las zonas de mayor tensión, sin embargo, son bastante fáciles de reparar, con el mismo material, compuesto tradicional, o con materiales fluidos?.

- Siempre necesitarán de una matriz o molde para su confección ${ }^{10}$.

- El cartucho de automezcla desperdicia material, el que queda en la cánula (de $0.5 \mathrm{I}$ a $\mathrm{I} .2 \mathrm{I}$ $g$ por cada mezclado según el producto).

- El costo elevado respecto a los MMA.

Dentro de las familias de los materiales bisacrílicos de reciente aparición, las generaciones de resinas se presentan con una alta resistencia a la compresión y a la flexión. Debido a su menor capa de inhibición de oxigeno y al nanorrelleno, no necesitan pulirse, siendo suficiente el ser limpiado con una gasa y alcohol. A su vez, la placa puede ser fácilmente removida. El resultado es una mejor higiene oral durante el tiempo que se usan las restauraciones provisionales, y una mayor comodidad y satisfacción para los pacientes. Este tipo de material resulta ser de fácil manipulación y con una alta resistencia mecánica ante los requerimientos funcionales $y$ brinda una 
textura con una gran estética. Una restauración provisional "proporciona protección, estabilización y función antes de la fabricación de la prótesis definitiva. También puede ser utilizado para determinar la eficacia estética, funcional y terapéutica de un plan de tratamiento. El pronóstico de una restauración fija depende de la calidad de esta restauración provisional".

En la actualidad se dispone en el mercado de diferentes marcas de resinas bis-acrílicas (Bis jet, Luxatemp Integridad, ProtempII, Protemp Garant, Protemp IV, Provitec, Smar Temp,Temphase, TurboTemp y Trim Ultra. temp, Luxatemp solar, Luxa-flow y DC Provipont)9las mismas se utilizan con cartuchos con pistola, así como también jeringas individuales (Cool temp. Coltene), estos sistemas de cartuchos de las resinas bis-acrílicas pueden garantizar una mezcla completa y homogénea carente de burbujas ${ }^{8}$.

Se trata de resinas en base de metacrilatos multifuncionales con relleno de vidrio y/o sílice.

\section{Caso Clínico}

Se presenta a la consulta una paciente, sexo femenino, de 36 años, con restauraciones defectuosas de carillas en los dos incisivos centrales superiores, cuyo principal motivo de consulta fue "mejorar la estética de sus dientes anteriores". Habiendo realizado el correspondiente diagnóstico y planteado el plan de tratamiento, para la rehabilitación de las dos piezas dentarias se procedió a la toma de impresión preliminar para la posterior confección de las coronas provisionales. A continuación se detalla el procedimiento realizado para la fabricación de las coronas provisorias con resina bis acrílica (Protemp IV) para la restauración de los incisivos centrales.

Se tomó una impresión preliminar con alginato y se obtuvo un modelo de estudio sobre el cual se realizó el encerado de las piezas dentarias I.I y 2.I (Fig. I). Luego se confeccionó una llave de silicona pesada sobre el modelo a modo de matriz. Se realizaron los recortes respectivos de tal manera de asegurar un tope por distal de cada pieza a provisionar (Fig. 2)

Se preparó la pistola dosificadora con el cartucho conteniendo el material para provisional autopolimerizable Protemp 4 y su correpondiente punta mezcladora (Fig. 3). Posteriormente se aplicó en

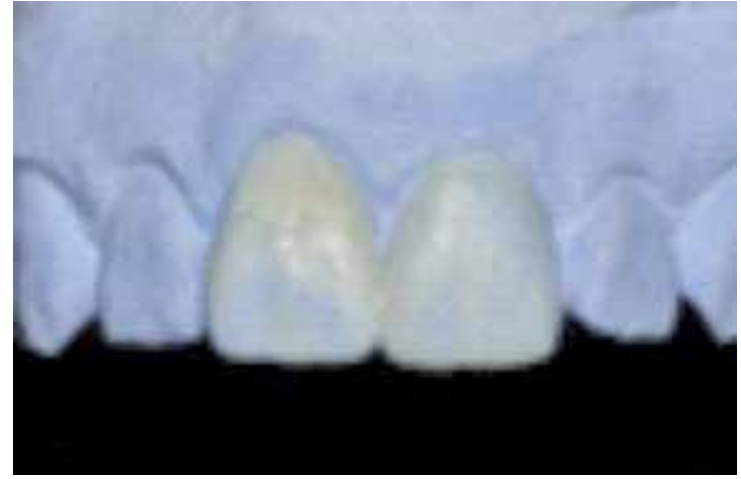

Figura I.

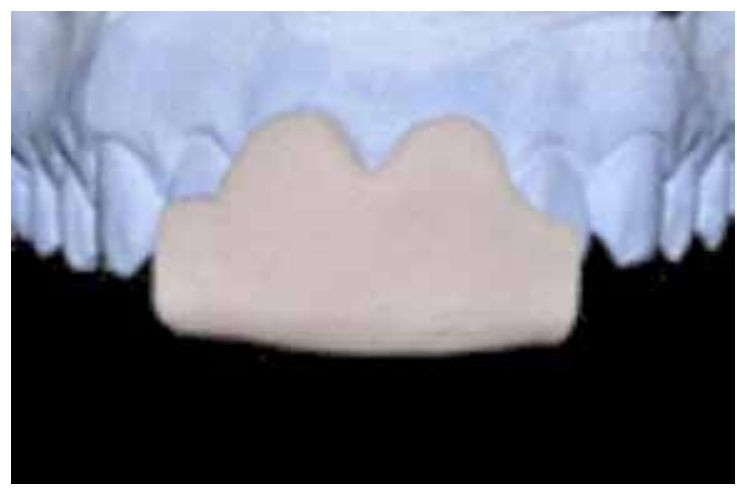

Figura 2.

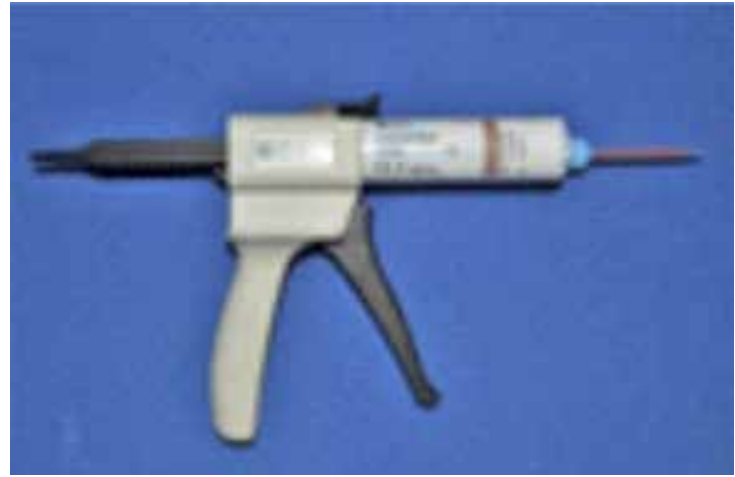

Figura 3.

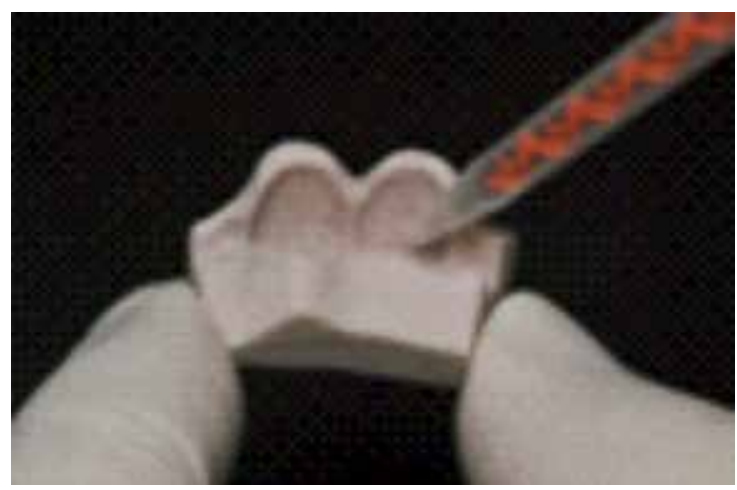

Figura 4. 
la matriz desechando una pequeña porción inicial y teniendo la precaución que la punta de la cánula se encuentre siempre sumergida en el material, para evitar la formación de burbujas de aire y en consecuencia defectos posteriores. (Fig. 4 y 5 ).

Se esperó el tiempo de polimerización inicial de un minuto y medio a dos y se retiró de la boca. A los cinco minutos de concluido el fraguado se retiró la matriz (Fig 6). Luego se recortaron los excesos y se eliminó la capa de resina superficial inhibida con una gasa embebida en alcohol (Fig 7). Se cementó la restauración provisoriamente con óxido de zinc sin eugenol (Fig 8). Para finalizar se realizaron los controles oclusales (Fig 9).

\section{Discusión}

Los requerimientos estéticos en cuanto a la estabilidad del color de las restauraciones provisionales, es una cualidad importante en la resina usada para provisional, particularmente para la reconstrucción de tratamientos durante un período largo ${ }^{13}$. Otra propiedad importante a destacar es la resistencia a la compresión y a la flexión, característica que presentan los materiales bisacrílicos de reciente aparición ${ }^{10}$.

Yannikakis y cols, ${ }^{13}$ compararon la estabilidad del color del metacrilato de metilo y la resina bis acrílica siendo la más estable la primera. Sin embargo Sham3 y cols encontraron una estabilidad de color aceptable en la resina bisacrilica (luxatemp) en comparación al metacrilato.

Koumjian J y D Firtel ${ }^{13}$ concluyeron que la resina de metacrilato de metilo autopolimerizable (cold pack) tenia menor estabilidad de color que el bis-acrílico compuesto (Protemp), mientras que otros dos metilmetacrilatos autopolimerizable (Truekit y Duralay) tenían más color estable que Protemp.

Las resinas acrílicas tie $\neg$ nen reacción exotérmica y contracción durante la polimerización y, al mismo tiempo, producen una alta cantidad de residuos de monómeros. Entre tanto, las resinas bisacrílicas tienen mejor estabilidad mecánica, son biocompatibles, sufren mínima reacción exotérmica durante la polimerización y no producen residuos de monómeros monofuncionales; por ello no causan irritación pulpar ni periodontal8. Young y col. compararon el bis-acrílico y el polimetacrilato en términos de oclusión, contorno,

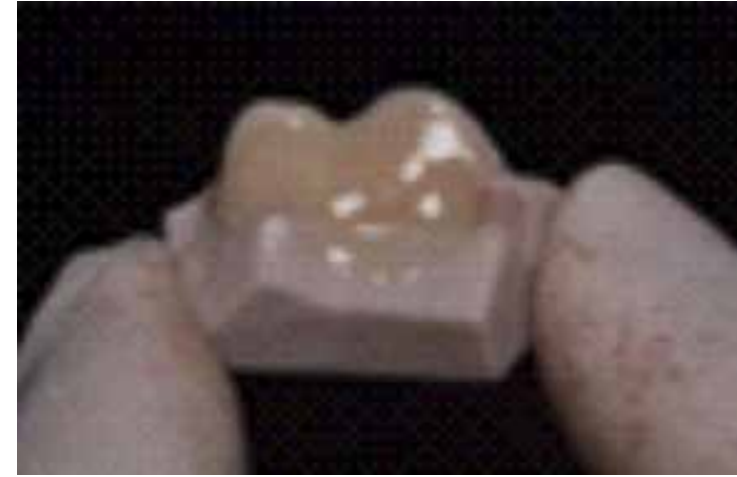

Figura 5.

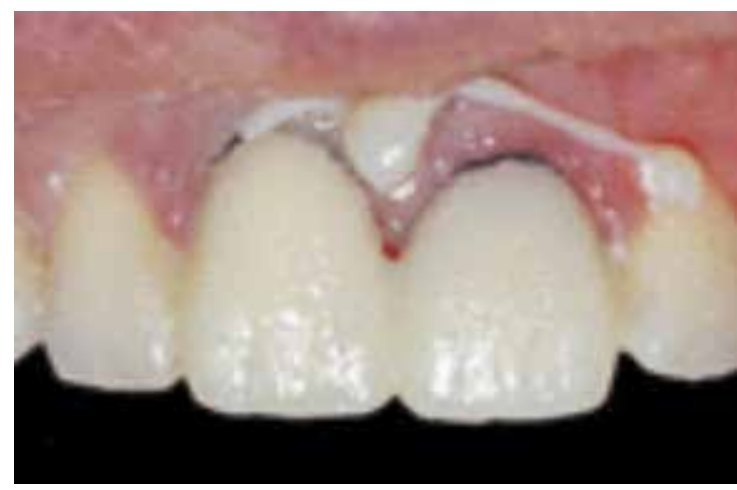

Figura 6.

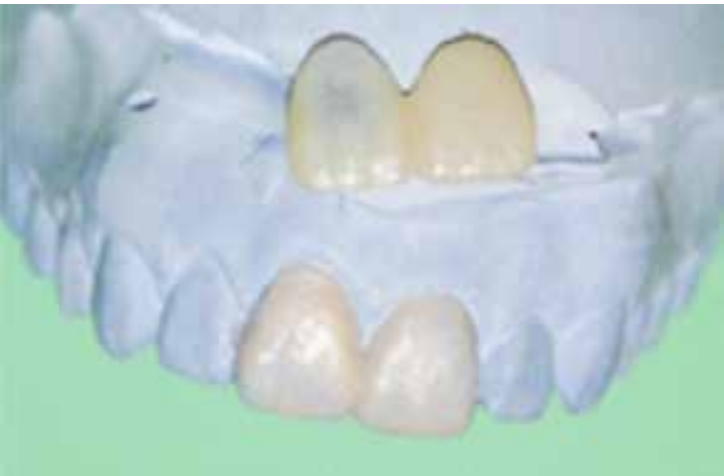

Figura 7.

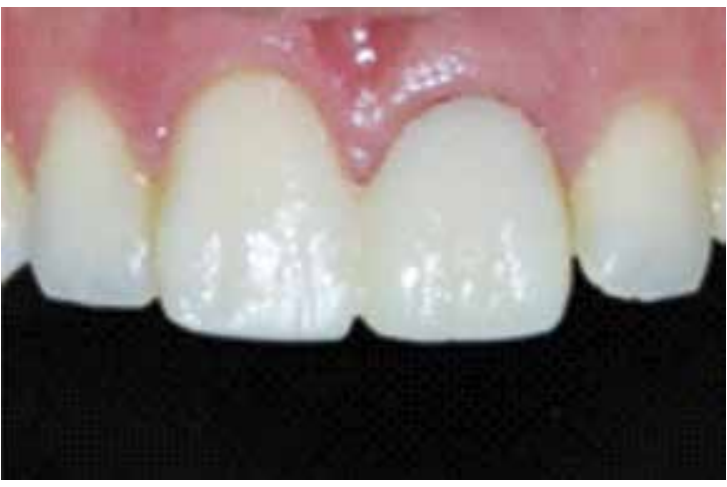

Figura 8 


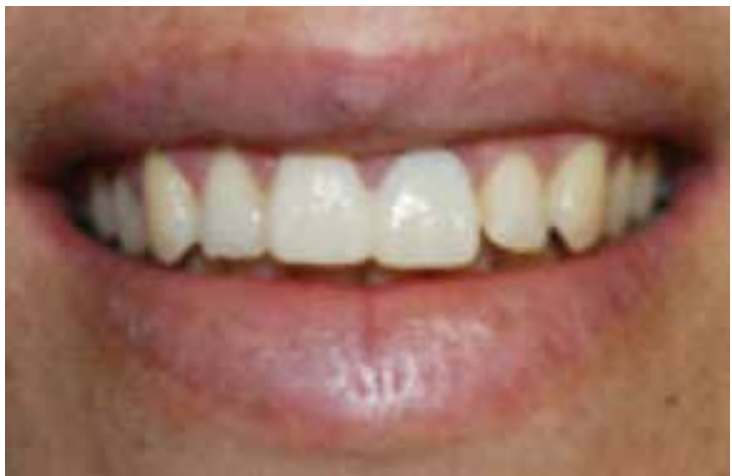

Figura 9.

fidelidad marginal, y el acabado, tanto en dientes anteriores como posteriores y encontraron que los materiales acrílicos bis-acrilicos son significativamente superior al PMMA en todas las categorías, a su vez estudios han concluido que Protemp IV es de color más estable y con propiedades mecánicas superiores ${ }^{10}$.

\section{Conclusión}

En la actuallidad los requerimientos de estética además de funcionalidad de las prótesis provisionales ha adquirido gran relevancia, lo que motivó a las casas comerciales a producir materiales con mejores propiedades que responden a estas demandas.

En el caso clínico presentado, las resinas bis acrílicas parecieran ser prometedoras, porque ofrecen algunas ventajas como ser el aumento de la seguridad y de la rapidez durante la preparación, su dosificación y automezcla, lo que facilita el procedimiento y permite la obtención de un provisional con una aceptable estética sin necesidad de pulido; si bien el costo de los cartuchos es más elevado.

Se puede conseguir muy buenos resultados con materiales en los que el método de aplicación (cartuchos) puede minimizar errores de preparación, desde el principio, facilitando así la confección con excelentes resultados.

\section{Bibliografía}

I. Carvajal H. J Prótesis fija. Preparaciones biológicas, impresiones y restauraciones provisionales. Ed. Mediterráneo 200I. Cap I2 Pagl I I-I I3 cap 13 II5-134, cap 14. I35-137

2. Givens EJ Jr, Neiva G, Yaman P, Dennison JB. Marginal adaptation and color stability of four provisional materials. J Prosthodont. 2008 Feb; 17(2): 97-I0I. Epub 2007 Oct 30.

3. Sham AS, Chu FC, Chai J, Chow TW. Color stability of pronvisional prosthodontic materials. J Prosthet Dent. 2004 May;9I(5): 447-52.

4. Doray PG, Wang X, Powers JM, Burgess JO. Accelerated aging affects color stability of provisional restorative materials. J Prosthodont. 1997 Sep; 6(3): 183-8.

5. Lang R, Rosentritt M, Leibrock A, Behr M, Handel G. Conlour stability of provisional crown and bridge restoration materials. $\mathrm{Br}$ Dent J. 1998 Nov; I85(9): 468-7I.

6. Guler, A, Yilmaz, F, Kunlunk T, Guler E, Kurt, S. Effects of different drinks on stainability of resin composite provisional. Journal of Prosthetic Dentistry. 2005 Agost: 94 (2): I I 8-124

7. Basi, A; Barrero C. Estudio in vitro para comprobar la estabilidad del color de materiales provisionales usados en prostodoncia. Dossier avances en materiales dentales Univ Odontol. 2011 JulDic; 30(65): 17-23. ISSN 0I 20-43I9.

8. Meyer A, Muller P. Prótesis Provisional directa. Quintenssence. 2007; 20 (9):559- 567.

9. Perry R , Magnuson B,. Provisional Materials: Compendium of Continuing Education in Dentistry.2012 33( 2): 59-62.

10. Krishna Prasad D, Manoj S., Harshitha A., Anupama Prasad D. provisional Restorations in Prosthodontic Rehabilitations-Concepts, Materials and Techniques. Nitte University Journal of Health Science June 20I2. 2 (2) :72-77

II. Ramkumar V, Sangeetha A, Kumar V. Efecto de la temperatura del agua en el ajuste de los márgenes de coronas provisionales durante la polimerización: estudio in vitro. J Pharm Sci Bioall 2012; 4:376-83.

12. Gaurav Gupta, Tina Gupta. Evaluación del efecto de diversas bebidas y de alimentos sobre la estabilidad del color de materiales provisionales. Estudio in vitro. J Dent Conserv. 20I I Jul-Sep; 14 (3): 287-292

13. Jalali H, Dorriz H, F Hoseinkhezri, Emadian Razavi SF. Estudio in vitro: la estabilidad del color de los materiales de restauración provisional. Indian J Dent Res 2012; 23:388-92. 
14. Tapia Silva $R$, Valenzuela Aránguiz $\mathrm{V}$, Zamorano Pino X, Baena Águila R., Cuantificación de la generación térmica en acrílicos de autopolimerización. Avance en Odontoestomatología. 2010; 26 (2): 91-96.

15. Stawarczyk B, Trottmann A y Fischer J. Prótesis provisionales. Quintessence técnica (ed. esp.). Febrero 2009.20 ( 2): 105-II2.

16. Cadafalch Cabaní J. Manual clínico de prótesis fija E. Cadafalch, Gabriel Edit harcourt brace. 1997; 63-67.

17. Bohnenkamp D., Garcia L. Repair of bis-acryl provisional restorations using flowable composite resin. The journal of prosthetic Dentistry. 2012; 92 (5): 500-502.

18. Fleisch L, Cleaton-Jones P, Forbes M, van Wyk J, Fat, C. Pulpalresponse to a bis-acryl- plastic (Protemp) temporary crown andbridge material. J Oral Pathol 1984;|3:622-631 